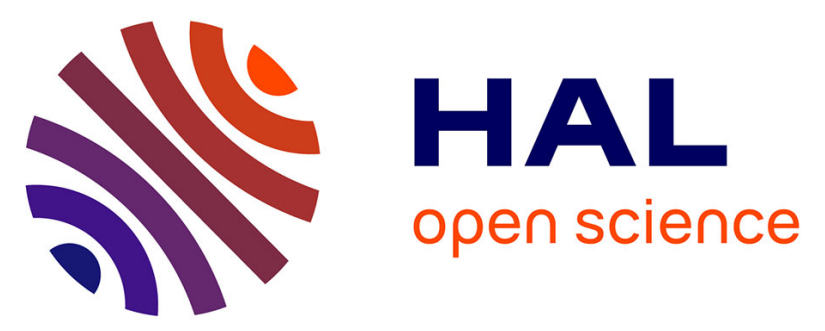

\title{
RADIATIVE ELECTRON CAPTURE BY FAST HIGHLY STRIPPED HEAVY IONS CHANNELED IN A THIN CRYSTAL
}

S. Andriamonje, M. Chevallier, C. Cohen, J. Dural, M. Gaillard, R. Genre, M. Hage-Ali, R. Kirsch, A. L'Hoir, B. Mazuy, et al.

\section{To cite this version:}

S. Andriamonje, M. Chevallier, C. Cohen, J. Dural, M. Gaillard, et al.. RADIATIVE ELECTRON CAPTURE BY FAST HIGHLY STRIPPED HEAVY IONS CHANNELED IN A THIN CRYSTAL. Journal de Physique Colloques, 1989, 50 (C1), pp.C1-285-C1-293. 10.1051/jphyscol:1989131 . jpa00229328

\section{HAL Id: jpa-00229328 https://hal.science/jpa-00229328}

Submitted on 1 Jan 1989

HAL is a multi-disciplinary open access archive for the deposit and dissemination of scientific research documents, whether they are published or not. The documents may come from teaching and research institutions in France or abroad, or from public or private research centers.
L'archive ouverte pluridisciplinaire HAL, est destinée au dépôt et à la diffusion de documents scientifiques de niveau recherche, publiés ou non, émanant des établissements d'enseignement et de recherche français ou étrangers, des laboratoires publics ou privés. 


\title{
RADIATIVE ELECTRON CAPTURE BY FAST HIGHLY STRIPPED HEAVY IONS CHANNELED IN A THIN CRYSTAL
}

\author{
S. ANDRIAMONJE, M. CHEVALLIER *, C. COHEN** J. DURAL ** " \\ M.J. GAILLARD*, R. GENRE*, M. HAGE-ALI*** 'R. KIRSCH*, A. L'HOIR** \\ B. MAZUY" , J. MORY****, J. MOULIN"*, J.C.'POIZAT', J.'REMILLIEUX", \\ D. SCHMAUS * and M. TOULEMONDE***
}

\author{
Centre D'Etudes Nucléaires de Bordeaux and IN2P3, F-33170 Gradignan, \\ France \\ * Institut de physique Nucléaire and IN2P3, Université Claude Bernard \\ Lyon-1, F-69622 Vilileurbanne Cedex, France \\ * Groupe de Physique des Solides de l'Ecole Normale supérieure, F-75251 \\ Paris Cedex 05, France \\ *** Centre Interdisciplinaire de Recherches avec les Ions Lourds, \\ F-14040 Caen Cedex, France \\ *** Groupe Physique Appliquée aux semiconducteurs, centre de Recherches \\ Nucleaires, F-67037 strasbourg Cedex, France \\ **** Laboratoire des Solides Irradiés, Ecole Polytechnique, F-91128 \\ Palaiseau Cedex, France
}

\begin{abstract}
Résumé - On sait que l'interaction des ions avec une cible monocristalline est très sensible à l'orientation du faisceau incident par rapport aux directions particulières du cristal. Nous avons montré que la canalisation d'ions lourds de très haute énergie modifie le ralentissement et l'échange de charge des ions. Non seulement les ions canalisés ne parcourent que des régions à faible densité électronique mais les électrons rencontrés ne peuvent être capturés au moyen du processus ordinaire non radiatif. Dans une expérience où des ions Xénon de charge $52^{+}, 53^{+}$et $54^{+}$sont envoyés sur un cristal mince de Silicium, nous observons que pour une incidence quelconque les ions transmis atteignent l'équilibre de charge mais que les ions canalisés sont pour la plupart transmis sans avoir changé d'état de charge. D'une part ils ne peuvent pas perdre d'électron et d'autre part le seul processus de capture qui leur est accessible est la capture radiative (REC), un processus d'ordinaire masqué par la prédominance de la capture mécanique (MEC). Nous avons observé les photons émis par REC et qui correspondent à des captures dans les couches $K, L$ ou $M$ des ions Xénon. $L a$ structure des raies observées reflète la distribution des vitesses des électrons capturés. La canalisation permet donc d'étudier la capture radiative par des ions de charge bien définie dans une cible dense d'électrons quasi-libres. Cette opportunité pourrait être étendue à d'autres processus concernant des ions lourds très rapides.
\end{abstract}

\begin{abstract}
Interaction of moving ions with single crystals is known to be very sensitive to the orientation of the incident beam with respect to the crystalline directions of the target. We have shown that channeling conditions strongly modify the slowing down and the charge exchange processes of high energy heavy ions. The reason is that channeled particles are prevented from approaching the target atoms, and then can interact only with loosely bound target electrons. This results not only in drastically reducing the electron density experienced by them, which lowers energy loss and electron loss, but also in inhibiting the radiationless capture of bound electrons, since most of the available electrons are quasi-free valence electrons. In an experiment where high energy $\times \mathrm{e}^{q+}$ ions, with $\mathrm{q}=52,53,54$, are directed onto a thin Si single crystal we observe that, whereas unchanneled ions reach charge equilibrium very rapidly, most channeled ions keep their incident charge state unchanged all along their passage through the crystal. On one hand the loss of their electron(s) $(q=52,53)$ is impossible. On the other hand the only way that is left to them to capture quasi-free electrons is the radiative electron capture (REC). This process does occurs also for unchanneled ions but is quite difficult to observe, particularly because of the overwhelming non radiative Mechanical Electron Capture(MEC). REC photons have been observed in channeling conditions and correspond to electron capture into the $K, L$ and $M$ shells of the projectiles. The shape of the REC photon lines reflects the momentum distribution of the electron encountered by channeled ions. Channeling offers a unique opportunity to study the radiative electron capture since it allows ions of well defined charge state to travel through a dense quasi-free electron target. This opportunity could be extended to other processes involving high energy heavy ions.
\end{abstract}




\section{1 - INTRODUCTION}

We describe an experimental work, performed at GANIL, in which the charge exchange process of highly stripped $25 \mathrm{MeV} / \mathrm{u}$ Xenon ions in a thin Silicon crystal is observed by means of two complementary methods, the measurement of the charge state distribution of transmitted ions and the spectrometry of the projectile X-rays emitted during the traversal of the target. In these experimental conditions the profound modification of the ion-solid interaction that is brought by channeling effects results first in the almost complete "freezing" of the incident charge state. This is due to the drastic reduction of electron loss and capture probabilities. Channeling conditions also reduce strongly the excitation probability of the projectiles, but the main consequence of channeling in our study lies in the emergence of the Radiative Electron Capture (REC), a process usually difficult to observe because it is overwhelmed, in amorphous targets (or unaligned crystals), by the Mechanical Electron Capture process (MEC). We recall first the general properties of channeling and the expected consequences on the interaction of such fast heavy ions with a solid target. Then, after a short description of the experimental set-up we describe our measurements of charge state distributions. Afterwards we describe the $X$-rays spectra in which ( $n p-1 s$ ) transitions and REC lines are observed, and discuss further developments of such studies.

\section{2 - CHANNELING IN BRIEF}

Channeling of positive ions in a crystal occurs when the direction of the incident beam is close to a plane or an axis direction of the crystal. Channeling properties are sketched in figure 1 where a positive ion enters a crystal in a parallel direction with an axis (a), with an impact parameter $\rho_{1}$ with respect to the atomic string. If $\rho_{1}$ is larger than the thermal vibration amplitude of the target atoms, the impact parameters of the successive collisions with the row atoms are not randomly distributed but are seen to increase slowly along the row, and the incident ion, repelled by the atomic string as a whole, is said to be channeled. The ion trajectory resulting from these correlated multiple scattering events can be calculated quite accurately by using the continuum potential approximation. The channeled projectiles are in a potential $V(\rho)$ that is the zero-order term of the Fourier series in which the periodic ion-atom potential at a distance $\rho$ along the string can be decomposed. The potential $V(\rho)$, represented in figure $\mathbf{1 b}$, would be infinite for $\rho=0$ if the target atoms were static. The thermal motion of target atoms can be shown to result in a finite value of $V(0)$.

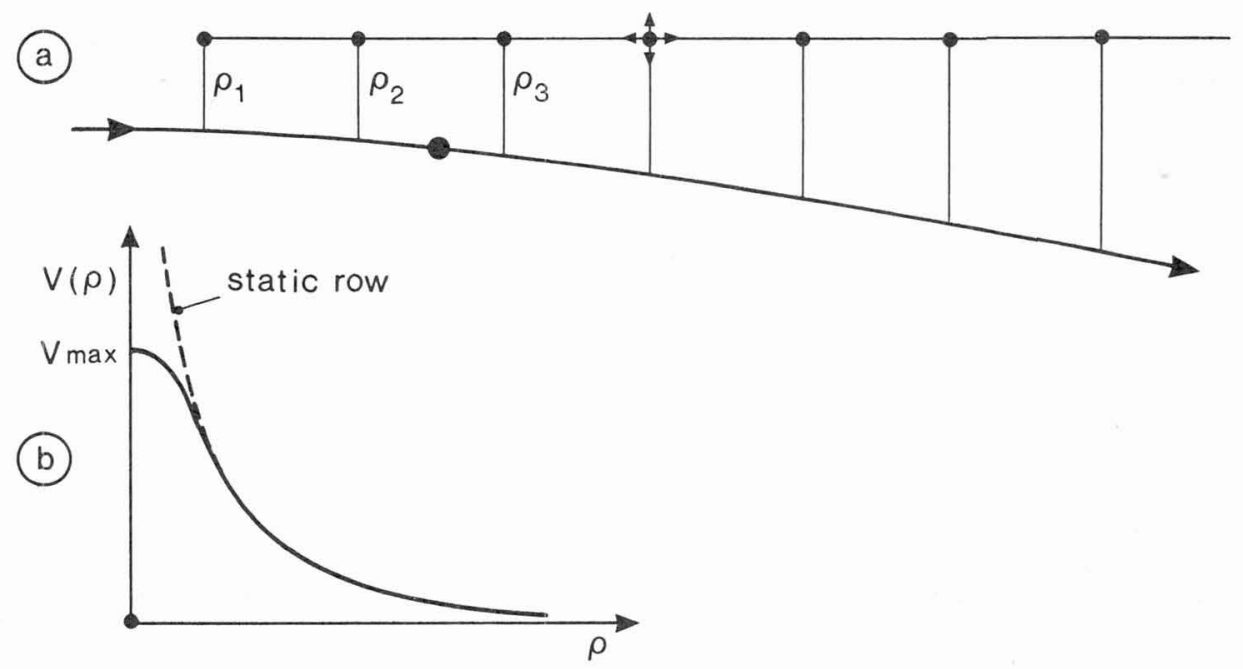

Fig. 1 - Channeling trajectory of a positive ion incident in a parallel direction with a crystal axis, with the impact parameter $\rho_{1}$. The arrows on one of the atoms of the string simulate the thermal motion of target atoms (a). Variation of the continuum potential with $\rho$, the distance to the axis, for a static row (dotted line) and with the thermal motion included (solid curve) (b). 
The entrance conditions, incidence angle to the axis direction and impact parameter, define the transverse energy of each projectile, that can be considered, in a first approximation, as being constant during the passage of the ion through a thin crystal. The value of the transverse energy determines the closest distant of approach to the atomic rows. The best channeled particles have the smallest transverse energy and then are maintained far from the atomic rows. The channeling critical angle $\Psi_{\text {, defined by }} E \Psi^{2}=V_{\text {max }}$, the potential maximum, is the maximum incidence angle to the axial direction for an ion to be channeled. This equation gives the energy dependence of the angular acceptance for channeling. (In the experimental conditions of this work, the critical angle was around 1 mradian).

In fact a more complete description of axial channeling must take more than one atomic string into account. On figure 2 we show the map of the target electron density averaged along the $<110>$ direction of a silicon crystal (a) and the map of the ion potential for a single charge ion, also averaged along the same direction (b). The electron density has been calculated by means of the electron wave functions in solid silicon and the potential has been deduced from the electron density. Along this axial direction, with the particular arrangement of the atomic rows in a diamond type crystal, the best channeled particles, with, for example, a transverse energy smaller than $1.5 \mathrm{eV}$ (contour 2 of figure $\mathbf{2 b}$ ) are confined within this contour and then between a given set of six atomic strings that is called a channel. Figure 2a shows that these particles encounter a very low electron density (less than $0.24 \mathrm{~A}^{-3}$ ) compared with the density encountered by a particle travelling randomly in silicon $\left(0.7 \mathrm{~A}^{-3}\right)$.

Then we can summarize the main properties of channeled ions : as they cannot approach the atomic rows, they do not interact closely with the target nuclei and with the innershell target electrons (in particular the suppression of Rutherford scattering is, from a practical point of view, the most useful feature of low energy channeling). Also the lowering of the encountered electron density reduces the energy loss rate by a factor up to two or three. The effects of channeling on the electron capture and loss of moving ions will be discussed later.

(a) mean electron density

(b) continuum potential

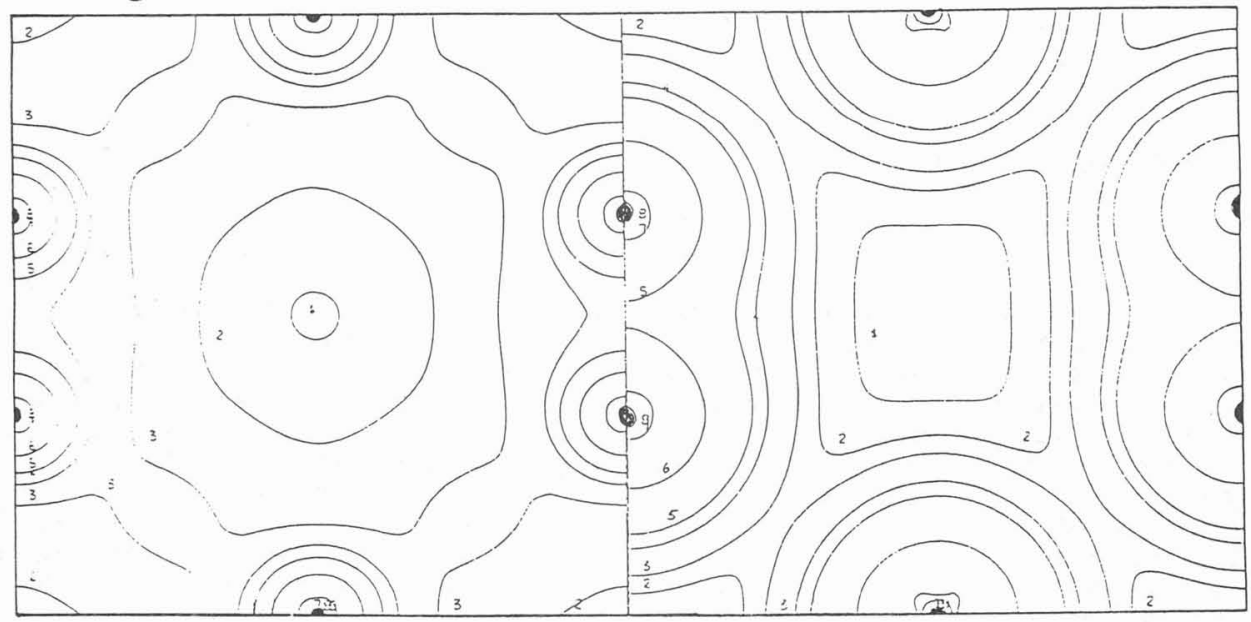

Fig. 2 - Map of the electron density in a silicon crystal, averaged along the $\langle 110\rangle$ direction. Contour lines 1 to $8: 0.032,0.1,0.24,0.5,1,3,10$ and $30\left(\mathrm{~A}^{-3}\right)(\mathrm{a})$.

Map of the continuum potential for a unit charge along the $\langle 110\rangle$ direction of Si. Contour lines 1 to $8: 0.5,1.5,2.5,7,20,50,100,200$ (eV) (b).

\section{3 - EXPERIMENTAL SET-UP}

The experimental arrangement, already described in a previous report $/ 2 /$, is schematized in figure 3. We used the LISE beam line at GANIL, a line specially designed to deliver highly stripped ion beams of excellent space and momentum definition. $25 \mathrm{MeV} / \mathrm{u} \mathrm{Xe}^{\mathrm{q}^{+}}$ions, with $\mathrm{q}=52,53$ or 54 , were produced by the post-stripping in a Be foil of the incident $27 \mathrm{MeV} \times \mathrm{e}^{35^{+}}$beam. The charge selection was operated by means of the magnetic spectrometer $M_{1}$ after tight collimation of the beam (angular divergence less than $0.2 \mathrm{mrad}$, diameter $4 \mathrm{~mm}$ ). Unfortunately, as the mean charge state of the beam transmitted through the Be foil was around 50, the beam intensity went down quite rapidly from $52^{+}$to $54^{+}$beams. After passage through a rotating beam-chopper used to 
monitor the beam intensity, either by the beam current measurement or by the counting in a Si-Li detector of the $X$-rays produced in the silver coating of the rotating blades, the beam was sent onto the crystal, a $17 \mu \mathrm{m}$ thick (111) Si crystal hold by a three-axis goniometer. The crystal was viewed by a solid state Ge detector positioned at $90^{\circ}$ to the beam. The transmitted beam was energy and charge analyzed by means of the magnetic spectrometer $M_{2}$ and a wire chamber. By stepping the intensity in $\mathrm{M}_{2}$ the energy loss spectrum associated to each charge state, and the charge state distribution could be obtained.

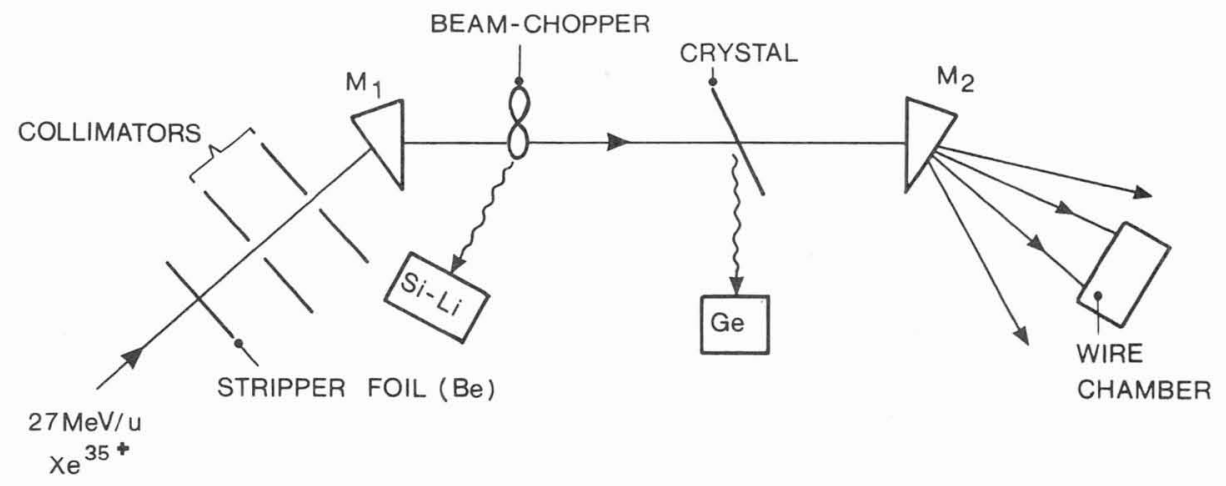

Fig. 3 - Experimental set-up.

\section{4 - CHARGE STATE MEASUREMENTS}

In figure 4 we show the charge state distributions obtained with $52^{+}, 53^{+}, 54^{+} \times e$ ions, respectively, incident along the $\langle 110\rangle$ direction of the Si crystal and also along a "random" direction, i.e. a direction free of channeling effects. In both cases the projectile pathlength through the crystal was $21 \mu \mathrm{n}$. The "random" charge distribution is measured to be the sane for the three incident charges, which means that the charge state equilibrium is reached. The average charge state is 49.5 . In this well known case a large number of capture and loss events have taken place. The dominant capture process is the Mechanical Electron Capture (MEC) of innershell target electrons, in which the incident ion, the captured electron and the ionized target atom share the available energy $/ 3 /$. The impact parameters are small, of the order the magnitude of the orbital of the electron to be captured. The Radiative Electron Capture (REC), in which the photon emission allows for energy and momentum conservation /4/ can also occur, but its probability is much smaller at this ion velocity although a much larger range of impact parameters contributes to REC (J.E. Miraglia, R. Gayet and J. Salin, private communication). As for the projectile electron loss from its $\mathrm{K}-$ and $\mathrm{L}$ shells it is due, as MEC, to low impact parameter collisions with target atoms.

On the contrary the three distributions obtained for axial alignment are quite different from each other, although of analogous shapes, and are then far from equilibrium. In each case the distribution is peaked on the incident charge and presents a rapid decrease on the low charge side. On the other hand all the $53^{+}$and $52^{+}$incident ions keep their(s) $\mathrm{K}$ electron(s). Moreover the detailed observation of the energy spectrum of transmitted ions of each particular charge state shows that ions "frozen" in their initial charge state were channeled in the crystal, as revealed by their reduced energy loss, which is 0.45 times the "normal" energy loss (in agreement with values measured with lighter ions at lower velocities). The ions that had captured one electron were channeled for a part of them only, whereas the rest of the transmitted ions were not channeled. Then the charge state distributions are easily understood : the high charge states correspond to channeled ions that have capture zero or one electron and the low charge states correspond to unchanneled ions that have experienced "random-type" charge exchange processes. It must be noted that the first observation of channeled "frozen" ions has been performed by Datz et al. /5/ with oxygen ions of much lower velocities transmitted through a thin gold crystal. In this case also the suppression of close collisions for channeled particles inhibits the MEC process, and then reduces strongly the electron capture probability since the only process available is REC. In our experiment the loss of $K$-shell 
electrons by channeled $X e$ ions is not only reduced but forbidden since collisions with a valence or an outershell target electron cannot transfer enough energy (even excitation is impossible, a feature that will be discussed later). Finally the probability for channeled ions of capturing an electron by REC is seen to decrease from the $54^{+}$case to the $52^{+}$case which is (qualitatively) understood because the REC into the $\mathrm{Xe} K$-shell is a large part of total REC, as also discussed below.

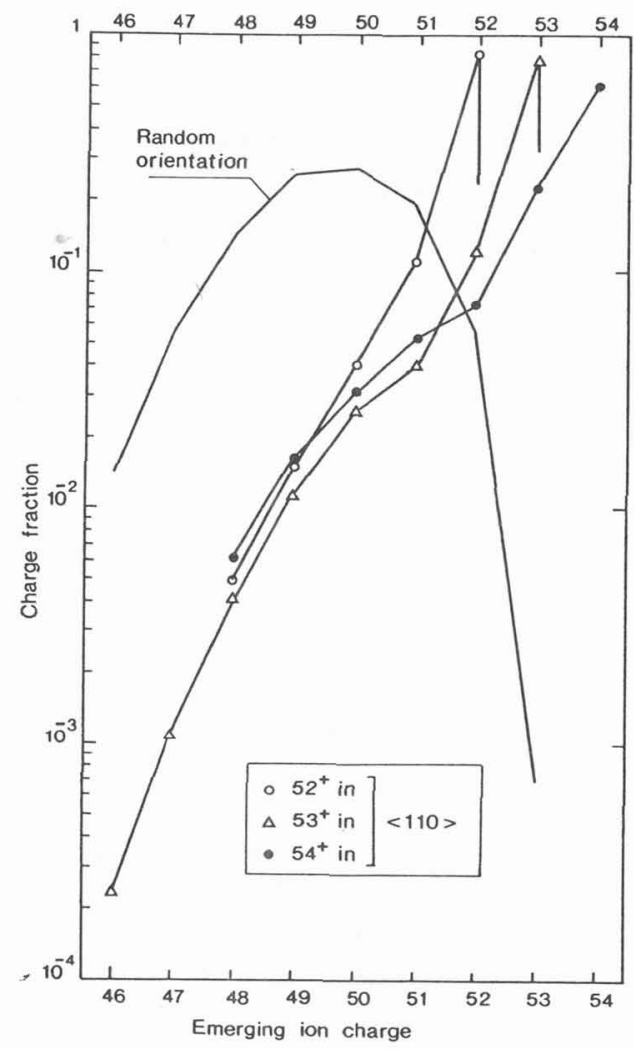

Fig. 4 - Charge state distributions obtained with $25 \mathrm{MeV} / \mathrm{u} 52^{+}, 53^{+}$or $54^{+}$Xe incident ions, in random conditions and for $\langle 110\rangle$ alignment after a pathlength of $21 \mu \mathrm{m}$ through the Si crystal.

\section{5 - STUDY OF X-RAY SPECTRA}

The first observation of REC by heavy ions in solids is due to Schnopper et al. /6/. The first experiment designed for the study of REC using channeling conditions was performed by the Oak Ridge group $/ 7 /$ in the wake of their previous work about channeling effects on charge exchange. However the experimental conditions (projectile atomic number and velocity) did not allow them to observe REC spectra in a very detailed way. The present experiment was then undertaken to yield a better observation of REC properties.

We recall the energetics of the radiative capture of a free electron assumed initially at rest by a moving ion. The photon emitted in the radiative capture into a state of binding energy $E_{l}$ has the energy $E_{R F C}=E_{l}+E^{\prime}$, where $E^{\prime}$ is the kinetic energy of an electron that would have the projectile velocity. 'This value is calculated in the ion rest frame but the corresponding energy of the photon detected at $90^{\circ}$ is quite close to this value.

The X-ray spectra of the solid state Ge detector viewing the crystal are shown in figures 5 to 7 for incident $52^{+}, 53^{+}$and $54^{+} \times e$ ions, respectively. In each case two spectra are given, for random and $\langle 110\rangle$ incidence, respectively, and for the same number of incident ions.

The information contained in these spectra is quite abundant, even for random incidence, that we consider first. 


\section{(a) "Random" spectra}

We start with the simplest case, the random spectrum obtained with $52^{+}$ions (figure 5 ), that will be used to describe the general features of this set of data. The spectrum is dominated by a series of lines that we find convenient to call the Lyman series $(\alpha, \beta, \gamma)$, although the ions are not $\mathrm{H}$-like ones. On the contrary, as we know that these $52^{+}$incident ions reach charge equilibrium in the target around an average value of 50, these lines result mainly from collisional excitation of $\mathrm{K}$-shell electrons. Note that the linewidths are larger than the energy resolution of the detector and are due to the slight charge dependence of the transition energies. Weaker lines are also observed, that correspond to the radiative electron capture into $K, L$, and $M$ shells of the projectile. The $L-$ and $M-$ REC peaks represent the contribution of REC to the electron capture necessary to reach the average equilibrium charge state of 50 , even if electron capture is dominated by MEC. As for the very weak K-REC peak, it corresponds to the REC contribution to the capture following collisional loss of a K-shell electron. Note that, as in all the presented spectra, the small peak at $18 \mathrm{keV}$ is due to an escape effect from the Ge detector in the detection of the Ly $\alpha$ photons.

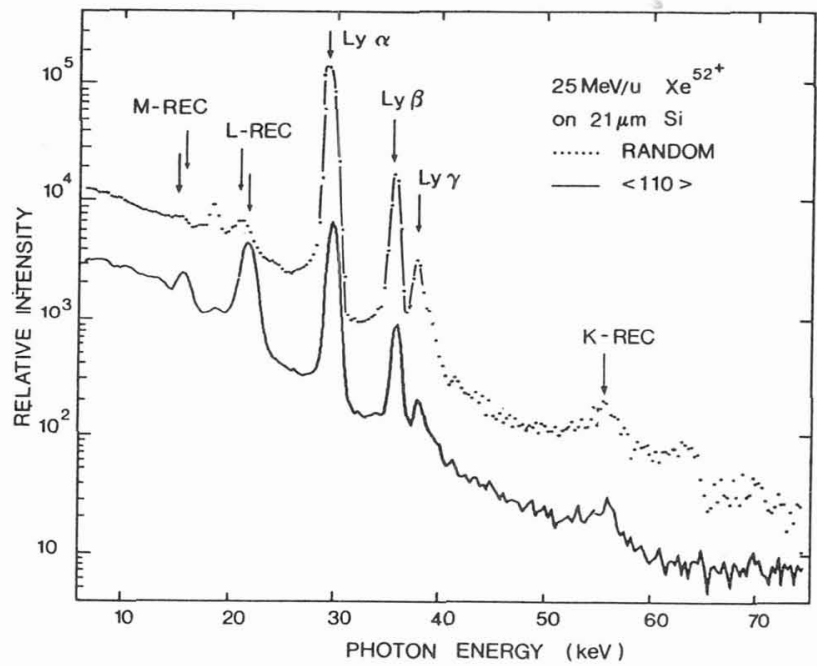

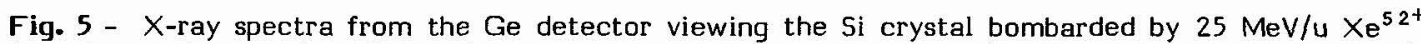
ions in random and $\langle 110\rangle$ alignment conditions, respectively (note the logarithmic scale).

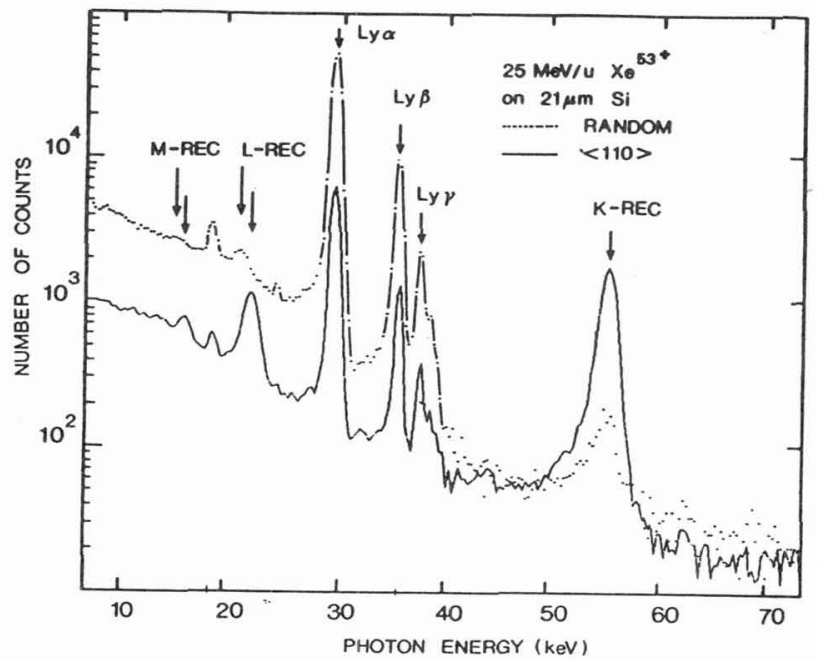

Fig. 6 - As for figure 5 with $53^{+}$ions. 
Lastly the strong continuum spectrum underlying the lines is in fact composed of two parts that cannot be distinguished. The first one is due to primary bremsstrahlung, the radiation emitted by target electrons accelerated by the Coulomb field of the projectile. This process can be considered as a radiative electron "capture" into the continuum of the projectile and the photon energy distribution decreases rapidly from zero to E', the low energy limit of the REC line series. The second one is due to secondary bremsstrahlung, i.e. the bremsstrahlung emission of the target electrons knocked on by the incident ions. The resulting photon energy distribution decreases also rapidly, but from zero to $\sim 4 \mathrm{E}^{\prime}$. There is unfortunately a third contribution that is due to nuclear background. This is negligible in the $52^{+}$case but not in the $53^{+}$and specially in the $54^{+}$case for which the beam intensities are lower.

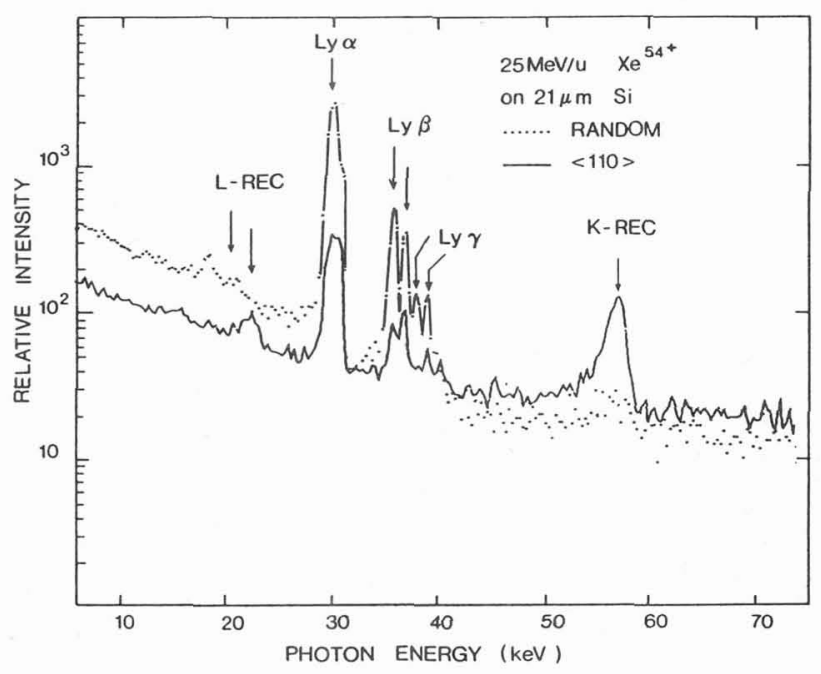

Fig. 7 - As for figure 5 with $54^{+}$ions.

If we consider now the random spectrum abtained with $53^{+}$ions (figure 6), why should it be different from the previous case ? The difference between the two situations is that the first charge exchange event will be, with a high probability, an electron capture, MEC or less probably REC. Afterwards, the two cases are identical because the crystal thickness is large compared with the mean pathlength between the entrance in the crystal and this first event. But we know from measurements from Meyerhof et al. /8/ and from predictions from Gayet and Salin (private communication) that in our experimental conditions the MEC process invalves mainly the excited states of $\mathrm{Xe}$ ions. Then (except for the $2 \mathrm{~s}$ state) these excited states decay very rapidly (in comparison with the projectile dwell time in the target) and contribute to the Lyman emission. In fact we observe experimentally that the comparison of the Lyman yield from $52^{+}$and $53^{+}$ions incoming along a random direction is compatible with the idea that an incident $53^{+}$ions produces one more Lyman photon in the target, on the average, than a $52^{+}$ion. It may be interesting to remark that the precise subtraction of the $52^{+}$spectrum from the $53^{+}$one would give the Lyman spectrum due to the electron capture by a $53^{+}$ion, which could be, more generally a method for studying capture without the necessity of using gas or very thin solid targets.

The last random spectrum, obtained with $54^{+}$ions (figure 7), is also, in an analog way, the sum of a $53^{+}$spectrum and of the Lyman spectrum associated with the electron capture by a bare $54^{+}$ion. The particularity of this spectrun is the fine structure appearing in the Lyman lines which is due to the higher energies of the $H$ - like Lyman transitions. In the case of $n=3 \rightarrow 1$ transitions for example this high energy line is due to the $3 p$ - is transition after the first electron capture by an incident $54^{+}$ion. If one supposes that the electron has been captured into the $3 p$ state (then ignoring cascade effects), the radiative H-like decay can be observed only if it occurs before a second electron capture takes place. If not, the photon will contribute to the lower energy peak of Lyß. This interesting competition between radiation decay and electron capture could be studied quantitatively but is complicated by cascade effects because the first electron can be captured into a higher level and decay through the $3 p$ state. We will come back to this point in the study of the 
spectra obtained in alignment conditions. A final remark about random spectra is that all of the incident ions have empty $L-$ and $M$ - shells. As a consequence the $L-$ and $M-R E C$ probabilities and then the number of L- and M- REC photons per incident ion should be the same in the three random spectra. We do observe that and deduce that the $54^{+}$Lyman spectrum is mainly due to the electron capture process.

\section{(b) "Aligned" spectra}

Although we have seen that in alignment conditions the incident ions present a broad distribution of transverse energies, we may simplify the discussion by distinguishing only between channeled and non channeled particles.

First of all one observes a strong reduction of the bremsstrahlung yield with respect to the random case. This is of course due to the reduction of the electron density encountered by channeled particles whereas the non channeled part of the beam experiences a normal electron density.

Another feature common to the three spectra is the strong reduction of the Lyman event probability. This is not surprising since both $K$ electron excitation and mechanical electron capture require small impact parameter collisions with target atoms. In the $52^{+}$case where the Lyman photons are due to excitation only, the channeled particles do not contribute at all and the channeling effect on the Lyman yield is stronger than the effect on bremsstrahlung. In the $53^{+}$and $54^{+}$cases, where Lyman transitions induced by the non channeled particles are also due to MEC, the channeled particles contribute by means of $M$ - and $L-$ REC (cf. below), and the channeling effect is less pronounced.

We note that the structures in the Lyman lines of the $54^{+}$random spectrum can be observed again, but it is quite intriguing that in both for $L y \alpha$ and $L y B$, the ratio between the two components is changed in favour of the $\mathrm{H}$ - like one. We believe that the competition between the deexcitation process of the first captured electron and the capture of second electron is different for particles incident along a random direction and for non channeled particles of an aligned beam : although random and non channeled particles may have the same average probability of close collision with target atoms in the crystal, the statistics of collisions is quite particular for non channeled particles, which spend short times in areas of high nuclear density (the atomic strings) and long times in areas of zero density (between atomic strings). This property of the unchanneled part of an aligned beam had never been described or observed before.

At last we come to the study of the REC photons, which form prominent lines. In the $52^{+}$spectrum, only $M$ - and L-REC lines show up whereas strong $K$ - REC peaks appear in the cases of incident $\mathrm{H}$-like $53^{+}$and bare $54^{+}$ions, as it could be expected.

The position of the peaks are well understood : the $M$ - and L- REC photons have higher energies than for random incidence and the shift increases with the incident charge. This is due to the charge state dependence of the binding energy of the L- shell electrons. In the same way the shift between the $53^{+}$and $54^{+} \mathrm{K}$ - REC peaks reflects the difference between the binding energies of the 1s electron(s) of $\mathrm{H}$ - like and He- like $X e$ ions.

The shape of the REC lines is also of great interest. In particular the K- REC peaks, located in an energy range where the continuum is low, appear rather wide and asymmetrical with a tail on the low energy side. The width is due to the momentum distribution (Compton profile) of the captured target electrons. The asymmetry results from the dependence of the REC cross section upon the relative velocity of the $\mathrm{Xe}$ ion and the electron to be captured. The shape indicates that the main contribution to REC comes from valence target electrons. Moreover, if one assumes that the angular distribution of K-REC photons follows a $\sin ^{2} \theta$ law, the K- REC intensity is found equal to 0.2 times the theoretical value deduced from Bethe and Salpeter estimates. This can be explained by the fact that the mean electron density experienced by channeled ions is calculated to be equal to 0.2 times the mean electron density in silicon.

On the other hand it is noticeable that the L- REC intensity is very little affected by channeling effects. Since the L- shell is empty in channeling conditions and nearly empty in random conditions, the steadiness of the L-REC intensity seems to indicate that the radiative electron capture into the $L$ - shell is not sensitive to the local electron density, a surprising result that further experiments should help to elucidate. More generally the study of the radiative electron capture into $(n>1)$ levels is complicated by the structure of these levels, both for the REC cross section and the photon angular distribution.

Further work on associated charge state measurements and $X$-ray observation under channeling conditions should include coincidence measurements, a study of photon angular distribution, a precise study of the crystal-beam orientation dependence of capture and excitation processes (previous results /2/ have not been discussed here). Other charge states could be used for the incident ions. 


\section{6 - CONCLUSION}

We have described the charge exchange processes involved in the interaction of helium-like, hydrogen-like and bare Xenon ions of high energy with a thin silicon single crystal. In particular we have shown that heavy ions channeled along a major axial direction interact mainly with quasi-free target electrons. Then in this case the ion-solid interaction becomes rather an ion-electron interaction and this type of target, equivalent to a dense electron target (in comparison with electron beams in crossed beam experiments), could be used for other studies.

One of them is the study of Resonance Electron Transfer (RTE), the inverse Auger process, in which the energy available in the capture of a quasi-free electron by a heavy ion is transfered into excitation of another electron of the ion. This resonant process could be studied in channeling conditions and yield then sharper resonances than in gas targets.

Another interesting possibility would be to use channeling conditions for the study of the electron loss process with incident ions carrying a large number of electrons.

A last and important remark is that the experimental situation described in this work is kinematically equivalent to the case of experimental works in which highly charged heavy ions, produced in an ion source and basically at rest, are bombarded by low-energy electrons, for instance for electron loss /9/ or excitation /10/ studies. The complementary of the two methods shoud be quite useful in the study of the interaction of highly charged ions with electrons.

We thank the GANIL staff for delivering to us a high quality beam. This work was specifically supported by CNRS and IN2P3, and partly supported by GDR 202.

\section{REFERENCES}

/1/ Gemmell D.S., Rev. Mod. Phys. 46 (1974) 129.

12/ Andriamonje S., Chevallier M., Cohen C., Dural J., Gaillard M.J., Genre R., Hage-Ali M., Kirsch R., L'Hoir A., Mazuy B., Mory J., Moulin J., Poizat J.C., Remillieux J., Schmaus $D$. and Toulemonde M., Phys. Rev. Lett. 59 (1987) 2271.

13/ Belkic D., Gayet R. and Salin A. Phys. Rep. 56 (1979) 279.

14/ Bethe H.A. and Salpeter E.E., Quantum Mechanics of one and two electron atoms (Academic Press, New York, N.Y.) 1957, p. 323.

15/ Datz S., Martin F.W., Moak, C.D., Appleton B.R. and Bridwell C.D., Rad. Eff. 12 (1972) 163.

16/ Schnopper H.W., Delvaille J.P., Kalate K., Sohval A.R., Abdulwahab M., Jones K.W. and Wegner H.E., Phys. Rev. Lett. 47 (1974) 61.

/7/ Appleton B.R., Ritchie R.H., Biggerstaff J.A., Noggle T.S., Datz S., Moak C.D., Verbeek M. and Neelavathi V.N., Phys. Rev. B19 (1979) 4347.

18/ Meyerhof W.E., Anholt R., Eichler J., Gould H., Munger Ch., Alonso J., Thieberger P. and Wegner H.E., Phys. Rev. A32 (1985) 3291.

19/ Donets E.D., Phys. Scripta T3 (1983) 11.

110/ Marss R.E., Levine M.A., Knapp D.A. and Henderson J.R., Phys. Rev. Lett. 60 (1988) 1715. 